Georgian Mathematical Journal

Volume 12 (2005), Number 1, 45-52

\title{
ON THE UNIQUENESS OF THE TWO-SIDED ERGODIC MAXIMAL FUNCTION
}

\author{
LASHA EPHREMIDZE
}

\begin{abstract}
It is proved that the two-sided ergodic maximal operator is oneto-one.

2000 Mathematics Subject Classification: 28D05.

Key words and phrases: Two-sided ergodic maximal function, uniqueness theorem.
\end{abstract}

1. Introduction. Let $(X, \mathbb{S}, \mu)$ be a finite measure space, $\mu(X)<\infty$, and let $T: X \rightarrow X$ be an invertible measure-preserving ergodic transformation.

For an integrable function $f, f \in L(X)$, the ergodic maximal function is denoted by $\mathbf{M}_{+} f$ (the subscript "+" indicates that the operator is right-sided):

$$
\mathbf{M}_{+} f(x)=\sup _{m \geq 0} \frac{1}{m} \sum_{k=0}^{m-1} f\left(T^{k} x\right), \quad x \in X .
$$

In [3] we prove that the ergodic maximal operator has the injectivity property, i.e. $\mathbf{M}_{+} f=\mathbf{M}_{+} g$ a.e. implies that $f=g$ a.e. (A different proof of this theorem is proposed in [4].) The same uniqueness theorem is proved for various onesided maximal operators in [1], [2]. As mentioned in the introduction of [3], the essential idea of proving these theorems is contained in the proof of the uniqueness theorem for the one-sided Hardy-Littlewood maximal operator but the problem remains still open for non-one-sided maximal operators. As an approach to the solution of this problem, in the present paper we propose the proof of the uniqueness theorem for two-sided ergodic maximal operator $\mathbf{M}$,

$$
\mathbf{M} f(x)=\sup _{n \leq 0<m} \frac{1}{m-n} \sum_{k=n}^{m-1} f\left(T^{k} x\right), \quad x \in X .
$$

Theorem 1. Let $f, g \in L(X)$ and

$$
\mathbf{M} f=\mathbf{M} g \text { a.e. }
$$

Then

$$
f=g \text { a.e. }
$$

An extension of the proof to the continuous case still requires to overcome some technical difficulties.

A simple example illustrates that the theorem is not valid for the symmetric ergodic maximal operator (see Section 5). The discrete nature of the operator plays a significant role in this situation (see [7]), and the continuous analogue of this theorem should in our opinion be correct. 
2. An auxiliary lemma. An analogue of the following lemma for the operator $\mathbf{M}_{+}$is proved in [3].

Lemma 1. Let $f \in L(X)$ and let

$$
F_{f}=\left\{x \in X: \mathbf{M} f(x)=\frac{1}{m-n} \sum_{k=n}^{m-1} f\left(T^{k} x\right) \text { for some } n \leq 0 \text { and } m>0\right\} \text {. }
$$

Then

$$
\mu\left(F_{f}\right)=\mu(X)
$$

and, consequently,

$$
\mu\left\{x \in X: T^{k} x \in F_{f} \quad \text { for all } k \in \mathbb{Z}\right\}=\mu(X) .
$$

Proof. Let

$$
\lambda_{0}=\frac{1}{\mu(X)} \int_{X} f d \mu
$$

The Individual Ergodic Theorem,

$$
\lim _{m \rightarrow \infty} \frac{1}{m} \sum_{k=0}^{m-1} f \circ T^{k}=\lambda_{0} \quad \text { a.e. }
$$

(see [6]) implies $\mu\left(\mathbf{M} f \geq \lambda_{0}\right)=\mu(X)$.

$$
\text { If } \begin{gathered}
\lim _{m \rightarrow \infty} \frac{1}{m} \sum_{k=0}^{m-1} f\left(T^{k} x\right)=\lambda_{0} \text { and } \lim _{n \rightarrow \infty} \frac{1}{n} \sum_{k=0}^{n-1} f\left(T^{-k} x\right)=\lambda_{0} \text {, then } \\
\lim _{n, m \rightarrow \infty} \frac{1}{n+m} \sum_{k=-n}^{m-1} f\left(T^{k} x\right)=\lambda_{0} .
\end{gathered}
$$

Thus (6) holds for a.a. $x \in X$, and this implies that a.a. $x \in\left(\mathbf{M} f>\lambda_{0}\right)$ belongs to $F_{f}$.

If $\mathbf{M} f(x)=\lambda_{0}$, then also $\mathbf{M}_{+} f(x)=\lambda_{0}$ and it is proved in [3] (see Corollary 1 therein) that for a.a. $x \in X$ there exist $m=m(x)$ such that $\frac{1}{m} \sum_{k=0}^{m-1} f\left(T^{k} x\right)=\lambda_{0}$. Consequently a.a. $x \in\left(\mathbf{M} f=\lambda_{0}\right)$ belongs to $F_{f}$.

The proof of (3) is completed.

Since $\left\{x \in X: T^{k} x \in F_{f}\right.$ for all $\left.k \in \mathbb{Z}\right\}=\bigcap_{k=-\infty}^{\infty} T^{k}\left(F_{f}\right)$, (4) holds as well.

3. Discrete maximal operator. Let $\Gamma$ denote the set of all two-sided sequences of real numbers indexed by integers $\mathbb{Z}$. The maximal operator $M$ is defined by

$$
M \alpha(q)=\sup _{n \leq q<m} \frac{1}{m-n} \sum_{k=n}^{m-1} \alpha(k), \quad q \in \mathbb{Z}, \quad \alpha \in \Gamma .
$$

Thus, if $\alpha(q)=f\left(T^{q} x\right)$, then

$$
M \alpha(q)=\mathbf{M} f\left(T^{q} x\right) .
$$


We consider the one-sided maximal operators $M_{+}$and $M_{-}$as well:

$$
M_{+} \alpha(q)=\sup _{m \geq q} \frac{1}{m-q+1} \sum_{k=q}^{m} \alpha(k) \text { and } M_{-} \alpha(q)=\sup _{n \leq q} \frac{1}{q-n+1} \sum_{k=n}^{q} \alpha(k) .
$$

Let us introduce some brief notations. Sets of the type $\{q \in \mathbb{Z}: M \alpha(q)>\lambda\}$, $\{x \in X: \mathbf{M} f(x)=\mathbf{M} g(x)\}, \ldots$ will be denoted by $(M \alpha>\lambda),(\mathbf{M} f=\mathbf{M} g), \ldots$

For $\alpha \in \Gamma$ and $I \subset \mathbb{Z}$, let $A_{I}=(1 / \operatorname{card}(I)) \sum_{k \in I} \alpha(k)$ and if $I_{p, r}=\{p$, $p+1, \ldots, r\}, p, r \in \mathbb{Z}, p \leq r$, is an interval of integers, then $A_{p, r}=A_{I_{p, r}}$.

We say that $I_{p, r}$ is a finite connected component of $N \subset \mathbb{Z}_{0}$ if $I_{p, r} \subset N$ and $p-1, r+1$ do not belong to $N$.

The proof of the following lemma is very easy but we formulate it for further reference.

Lemma 2. Let $I, J \subset \mathbb{Z}$ be disjoint and $K=I \cup J$.

(i) If $A_{K}=\lambda$ and $A_{J}=\lambda$, then $A_{I}=\lambda$;

(ii) If $A_{K}=\lambda$ and $A_{J}<\lambda$, then $A_{I}>\lambda$;

(iii) If $A_{K}>\lambda$ and $A_{J} \leq \lambda$, then $A_{I}>\lambda$;

(iv) If $A_{I}=\lambda$ and $A_{J}>\lambda$, then $A_{K}>\lambda$.

Proof. If $\sum_{k \in K} \alpha(k)=\lambda \operatorname{card}(K)$ and $\sum_{k \in J} \alpha(k)=\lambda \operatorname{card}(J)$, then $\sum_{k \in I} \alpha(k)=$ $\lambda(\operatorname{card}(K)-\operatorname{card}(J))=\lambda \operatorname{card}(I)$ and (i) follows.

If $\sum_{k \in I} \alpha(k) \leq \lambda \operatorname{card}(I)$ and $\sum_{k \in J} \alpha(k)<\lambda \operatorname{card}(J)$, then $\sum_{k \in K} \alpha(k)<\lambda(\operatorname{card}(I)+$ $\operatorname{card}(J))=\lambda \operatorname{card}(K)$ which is a contradiction and (ii) follows.

In a similar way one can show (iii) and (iv).

For $\alpha \in \Gamma$, let $N_{\alpha} \subset \mathbb{Z}$ be the set of integers for which the supremum is achieved after finitely many steps, i.e., $q \in N_{\alpha}$ if and only if $M \alpha(q)=A_{n, m}$ for some $n, m \in \mathbb{Z}, n \leq q \leq m$. Observe that if $\alpha_{x}(k)=f\left(T^{k} x\right), f \in L(X)$, then $k \in N_{\alpha_{x}} \Leftrightarrow T^{k} x \in F_{f}$. Hence, for a.a. $x \in X$, we have (see (4))

$$
N_{\alpha_{x}}=\mathbb{Z}
$$

Lemma 3. Let $\alpha \in \Gamma, q \in N_{\alpha}$ and

$$
M \alpha(q)=\lambda,
$$

and let $I_{p, q-1}$ and $I_{q+1, r}$ be finite connected components of $\left(M_{-} \alpha>\lambda\right)$ and $\left(M_{+} \alpha>\lambda\right)$, respectively, then

$$
M \alpha(q)=\frac{1}{r-p+1} \sum_{k=p}^{r} \alpha(k)=\lambda .
$$

We assume that if $M_{-} \alpha(q-1) \leq \lambda$ (i.e., $I_{p, q-1}=\varnothing$ ), then $p=q$ and if $M_{+} \alpha(q+1) \leq \lambda$ (i.e., $\left.I_{q+1, r}=\varnothing\right)$, then $r=q$ in (9) and in the proof below.

Proof. Let

$$
\lambda=M \alpha(q)=\frac{1}{q_{2}-q_{1}+1} \sum_{k=q_{1}}^{q_{2}} \alpha(k),
$$


where $q_{1} \leq q \leq q_{2}$. We can assume that $I_{q_{1}, q_{2}}$ is minimal in a sense that it contains no proper subset $I_{p_{1}, p_{2}} \ni q$ for which $A_{p_{1}, p_{2}}=\lambda$, and we will show that $q_{1}=p$ and $q_{2}=r$.

If $q_{2}>r$, then $A_{r+1, q_{2}} \leq \lambda$, since $r+1 \notin M_{+} \alpha>\lambda$. If now

$$
A_{r+1, q_{2}}=\lambda
$$

then Lemma 2 (i), (10) and (11) imply that $A_{q_{1}, r}=\lambda$, which contradicts the minimality of $I_{q_{1}, q_{2}}$, and if

$$
A_{r+1, q_{2}}<\lambda
$$

then Lemma 2 (ii), (10) and (12) imply that $A_{q_{1}, r}>\lambda$, which is a contradiction, since $q \in I_{q_{1}, r}$ and (10) holds.

Analogously, $q_{1}$ cannot be smaller than $p$.

If $q_{2}<r$, then

$$
A_{q_{2}+1, r}>\lambda
$$

(see [3], Lemma 4) and Lemma 2 (iv), (10) and (13) imply that $A_{q_{1}, r}>\lambda$, which is a contradiction, since $q \in I_{q_{1}, r}$ and (10) holds.

Analogously, $q_{1}$ cannot be larger than $p$.

Lemma 4. Let $\alpha \in \Gamma$ and let $I_{p, r}$ be a finite connected component of ( $M \alpha>$ $\left.\lambda_{0}\right)$ for some $\lambda_{0}<\infty$. Then for each $q \in I_{p, r}$ there exists an interval of integers $J \subset I_{p, r}$ containing $q$ such that $M \alpha(q)=A_{J}$.

Proof. Let $\epsilon<M \alpha(q)-\lambda_{0}$ and $A_{q_{1}, q_{2}}>\lambda>M \alpha(q)-\epsilon$, where $q_{1} \leq q \leq q_{2}$. If $r<q_{2}$, then $A_{r+1, q_{2}} \leq \lambda$ since $r+1 \notin\left(M \alpha>\lambda_{0}\right)$ and we can apply Lemma 2 (iii) to conclude that $A_{q_{1}, r}>\lambda$. Similarly, we can deal with $q_{1}$ and it follows that for each $\epsilon>0$ there exists an interval $I \subset I_{p, r}$ containing $q$ such that $A_{I} \geq M(q)-\epsilon$. Consequently, $M(q)=A_{J}$ for some interval $J \subset I_{p, r}, J \ni q$, since the number of such intervals is finite and the lemma follows.

Lemma 5. Let $\alpha \in \Gamma$, let $I_{p, r}$ be a finite connected component of $\left(M \alpha>\lambda_{0}\right)$ for some $\lambda_{0}<\infty$, and let $\lambda \geq \lambda_{0}$. If we know the values of $\alpha$ on $(M \alpha>\lambda) \cap I_{p, r}$, then we can identify the sets $\left(M_{-} \alpha>\lambda\right) \cap I_{p, r}$ and $\left(M_{+} \alpha>\lambda\right) \cap I_{p, r}$.

Proof. Obviously, $\left(\left(M_{+} \alpha>\lambda\right) \cap I_{p, r}\right) \subset\left((M \alpha>\lambda) \cap I_{p, r}\right)$. Thus we should determine for each $q \in\left((M \alpha>\lambda) \cap I_{p, r}\right)$ whether it belongs to $\left(M_{+} \alpha>\lambda\right) \cap I_{p, r}$. Assume $s \geq q$ is the minimal integer outside $(M \alpha>\lambda)$ (note that $s \leq r$ and we know all values of $\alpha$ on $\left.I_{q, s-1}\right)$. If now $q_{2} \geq s$ is such that $A_{q, q_{2}}>\lambda$, then $A_{s, q_{2}} \leq \lambda$ and $A_{q, s-1}>\lambda$ because of Lemma 2 (iii). Hence $q \in\left(\left(M_{+} \alpha>\lambda\right) \cap I_{p, r}\right)$ if and only if $\sup _{q_{2} \in I_{q, s-1}} A_{q, q_{2}}>\lambda$.

In a similar way one can identify $\left(M_{-} \alpha>\lambda\right)$.

Lemma 6. Let $\alpha \in \Gamma$ and let $I_{p, r}$ be a finite connected component of ( $M \alpha>$ $\lambda$ ) for some $\lambda<\infty$. Then the values $M \alpha(q), q \in I_{p, r}$, uniquely define the values $\alpha(q), q \in I_{p, r}$. Thus if $M \alpha(q)=M \beta(q), q \in \mathbb{Z}$, for some $\beta \in \Gamma$, then $\alpha(q)=\beta(q), q \in I_{p, r}$. 
Proof. Note that $q \in N_{\alpha}$ for each $q \in I_{p, r}$ because of Lemma 4.

Set the values $M \alpha(q), q \in I_{p, r}$, in descending order, i.e., assume $\lambda_{1}>\lambda_{2}>$ $\cdots>\lambda_{j}>\lambda$, where

$$
I_{i}=\left\{q \in I_{p, r}: M \alpha(q)=\lambda_{i}\right\} \neq \varnothing
$$

and

$$
\bigcup_{i=1}^{j} I_{i}=I_{p, r}
$$

Define the values $\alpha(q)$ by induction with respect to $i$. For $i=1, \alpha$ is equal to $\lambda_{1}$ on $I_{1}$, i.e.,

$$
\alpha(q)=\lambda_{1}
$$

for all $q \in I_{1}$. Indeed, it follows from Lemma 3 that $M \alpha(q)=\alpha(q)$ for each $q \in I_{1}$, since $q-1$ and $q+1$ do not belong to $\left(M \alpha>\lambda_{1}\right)$.

Let us now assume that $\alpha$ is already defined on $I_{1} \cup I_{2} \cup \cdots \cup I_{i}, i<j$; we will define it on $I_{i+1}$.

For $q \in I_{i+1}$ (which implies that $\left.M \alpha(q)=\lambda_{i+1}\right)$, since $\left(\left(M \alpha>\lambda_{i+1}\right) \cap I_{p, r}\right)=$ $I_{1} \cup I_{2} \cup \cdots \cup I_{i}$ and we know the values of $\alpha$ on this set, we can identify $\left(M_{-} \alpha>\lambda_{i+1}\right) \cap I_{p, r}$ and $\left(M_{+} \alpha>\lambda_{i+1}\right) \cap I_{p, r}$, because of Lemma 5 .

Consequently, we can apply Lemma 3 and all the values in equation (9) are known except $\alpha(q)$ which can be determined.

Corollary 1. Let $\alpha \in \Gamma$ and let $M \alpha(p)<M \alpha\left(q_{0}\right)>M \alpha(r)$ for some $p, r \in \mathbb{Z}, p \leq q_{0} \leq r$. Then the values $M \alpha(q), q \in \mathbb{Z}$, uniquely define the value $\alpha\left(q_{0}\right)$. Thus if some other $\beta \in \Gamma$ is given such that $M \alpha(q)=M \beta(q), q \in \mathbb{Z}$, then $\alpha\left(q_{0}\right)=\beta\left(q_{0}\right)$.

Proof. If we take $\lambda$ strictly between $M\left(q_{0}\right)$ and $\max ((M \alpha(p), M \alpha(r))$, then there is a finite connected component of $(M \alpha>\lambda)$ containing $q_{0}$.

Lemma 7. Let $\alpha \in \Gamma$, let $q \in N_{\alpha}$ and $M \alpha(q) \geq \lambda_{0}$ for each $q \in \mathbb{Z}$, and let

$$
\operatorname{card}\left\{k \geq 0: \alpha(k)=\lambda_{0}\right\}=\operatorname{card}\left\{k \leq 0: \alpha(k)=\lambda_{0}\right\}=\infty .
$$

Then the values $M \alpha(q), q \in \mathbb{Z}$, uniquely define the values $\alpha(q), q \in \mathbb{Z}$. Thus if $M \alpha(q)=M \beta(q), q \in \mathbb{Z}$, for some $\beta \in \Gamma$ such that $q \in N_{\beta}$ and $M \beta(q) \geq \lambda_{0}$ for each $q \in \mathbb{Z}$ and

$$
\operatorname{card}\left\{k \geq 0: \beta(k)=\lambda_{0}\right\}=\operatorname{card}\left\{k \leq 0: \beta(k)=\lambda_{0}\right\}=\infty,
$$

then $\alpha(q)=\beta(q), q \in \mathbb{Z}$.

Proof. Relation (14) implies that the set $\left(M \alpha>\lambda_{0}\right)$ consists of finite connected components. Hence we can determine the values $\alpha(q), q \in\left(M \alpha>\lambda_{0}\right)$, by Lemma 6 . It also follows from Lemma 5 that we can identify finite connected components of $\left(M_{+} \alpha>\lambda_{0}\right)$ and $\left(M_{-} \alpha>\lambda_{0}\right)$, and if $M \alpha(q)=\lambda_{0}$, then we can use Lemma 3 to find the only unknown quantity $\alpha(q)$ of equation (9). 
4. Proof of Theorem 1. Equation (1) implies that $\mathbf{M} f\left(T^{k} x\right)=\mathbf{M} g\left(T^{k} x\right)$, $k \in \mathbb{Z}$, for a.a. $x \in X$ (more exactly, for all $x \notin \bigcap_{k=-\infty}^{\infty} T^{k}(\mathbf{M} f \neq \mathbf{M} g)$ ). Thus

$$
M \alpha_{x}(k)=M \beta_{x}(k), \quad k \in \mathbb{Z},
$$

for a.a. $x \in X$, where

$$
\alpha_{x}(k)=f\left(T^{k} x\right) \quad \text { and } \quad \beta_{x}(k)=g\left(T^{k} x\right)
$$

(see (7)), and we will show that

$$
\alpha_{x}(k)=\beta_{x}(k), \quad k \in \mathbb{Z},
$$

for a.a. $x \in X$, which completes the proof of (2).

Relation (8) implies that, for a.a. $x \in X$,

$$
\mathbb{Z}=N_{\alpha_{x}}=N_{\beta_{x}} .
$$

Let $\lambda_{0}$ be ess inf $\mathbf{M} f=\operatorname{ess} \inf \mathbf{M} g$. Then

$$
\mathbf{M} f\left(T^{k} x\right)=\mathbf{M} g\left(T^{k} x\right) \geq \lambda_{0}, \quad k \in \mathbb{Z},
$$

for a.a. $x \in X$ (for all $x \notin \bigcap_{k=-\infty}^{\infty} T^{k}\left(\mathbf{M} f=\mathbf{M} g<\lambda_{0}\right)$ and, consequently,

$$
M \alpha_{x}(k)=M \beta_{x}(k) \geq \lambda_{0}, \quad k \in \mathbb{Z},
$$

for a.a. $x \in X$.

We consider two cases:

(i) $\mu\left(\mathbf{M} f=\lambda_{0}\right)=\mu\left(\mathbf{M} g=\lambda_{0}\right)=0$. Then

$$
M \alpha_{x}(k)=M \beta_{x}(k)>\lambda_{0}, \quad k \in \mathbb{Z},
$$

for a.a. $x \in X$.

Choose any decreasing sequence $\lambda_{i}, i=1,2, \ldots$, convergent to $\lambda_{0}$. Then

$$
\mu\left(\mathbf{M} f<\lambda_{i}\right)=\mu\left(\mathbf{M} g<\lambda_{i}\right)>0, \quad i=1,2, \ldots,
$$

and the Individual Ergodic Theorem implies that

$$
\begin{aligned}
\operatorname{card}\left\{k \leq 0: T^{k} x\right. & \left.\in\left(\mathbf{M} f<\lambda_{i}\right)\right\} \\
& =\operatorname{card}\left\{k \geq 0: T^{k} x \in\left(\mathbf{M} g<\lambda_{i}\right)\right\}=\infty, \quad i \geq 1,
\end{aligned}
$$

for a.a. $x \in X$. Now, for each $x$ satisfying (15), (19) and (20) and for any $q_{0} \in \mathbb{Z}$, since $M \alpha_{x}\left(q_{0}\right)>\lambda_{0}$, there exist $p \leq q_{0}$ and $r \geq q_{0}$ such that $M \alpha_{x}(p)<$ $M \alpha_{x}\left(q_{0}\right)>M \alpha_{x}(r)$. Thus we can apply Corollary 1 of Lemma 6 to conclude that

$$
\alpha_{x}\left(q_{0}\right)=\beta_{x}\left(q_{0}\right)
$$

and, since $q_{0}$ is an arbitrary integer, (16) is proved.

(ii) $\mu\left(\mathbf{M} f=\lambda_{0}\right)=\mu\left(\mathbf{M} g=\lambda_{0}\right)>0$. Then, by the Individual Ergodic Theorem,

$$
\operatorname{card}\left\{k \leq 0: T^{k} x \in\left(\mathbf{M} f=\lambda_{0}\right)\right\}=\operatorname{card}\left\{k \geq 0: T^{k} x \in\left(\mathbf{M} g=\lambda_{0}\right)\right\}=\infty
$$

and

$$
\operatorname{card}\left\{k \leq 0: M \alpha_{x}(k)=\lambda_{0}\right\}=\operatorname{card}\left\{k \geq 0: M \alpha_{x}(k)=\lambda_{0}\right\}=\infty
$$


for a.a. $x \in X$. If now $x$ satisfies (15), (17), (18) and (21), then we can apply Lemma 7 and establish the validity of (16).

5. Counterexample for the symmetric maximal operator. For a measurable function $f$, the symmetric ergodic maximal function $\mathbf{M}_{s} f$ is defined by

$$
\mathbf{M}_{s} f(x)=\sup _{m \geq 0} \frac{1}{2 m+1} \sum_{k=-m}^{m} f\left(T^{k} x\right), \quad x \in X .
$$

Let the measure space $X$ be $\{0,1,2\}$ with counting measure $\mu$ and let $T$ be the transformation $T(x)=x+1(\bmod 3)$. Define the functions $f$ and $g$ as follows: $f(x)=x, x \in X$ and $g(0)=1, g(1)=0, g(2)=2$. Then $\mathbf{M}_{s} f(0)=\mathbf{M}_{s} g(0)=$ $\mathbf{M}_{s} f(1)=\mathbf{M}_{s} g(1)=1$ and $\mathbf{M}_{s} f(2)=\mathbf{M}_{s} g(2)=2$, i.e., $\mathbf{M}_{s} f=\mathbf{M}_{s} g$, while $f \neq g$.

6. Infinite measure case. For infinite measure spaces, $\mu(X)=\infty$, the uniqueness theorem is not any longer valid. For example, every integrable negative function $f$ has the maximal function $\mathbf{M} f$ equal identically to 0 since the limit of ergodic averages of every integrable function converges to 0 almost everywhere,

$$
\lim _{m \rightarrow \infty} \frac{1}{m} \sum_{k=0}^{m-1} f\left(T^{k} x\right)=0
$$

for a.a. $x \in X, f \in L(X)$ (see [5]).

However, for non-negative functions, the uniqueness theorem is correct:

Theorem 2. Let $T$ be an invertible measure-preserving ergodic transformation of a $\sigma$-finite measure space $(X, \mathbb{S}, \mu)$. If $0 \leq f, g \in L$ and $\mathbf{M} f=\mathbf{M} g$ almost everywhere, then $f=g$ almost everywhere.

This theorem can be proved in the same way as for the one-sided maximal operator $\mathbf{M}_{+}$in Section 4 of [3]. Moreover, the exact analog of Theorem 2 in [3] is correct for the two-sided operator $\mathbf{M}$.

Theorem 3. Let $T$ be an invertible measure-preserving ergodic transformation of a $\sigma$-finite measure space $(X, \mathbb{S}, \mu)$ with $\mu(X)=\infty$.

(i) If $f \in L$ and

$$
\mathbf{M} f=\mathbf{M} g \text { a.e. on } X,
$$

then $f=g$ a.e. on $(\mathbf{M} f>0)$;

(ii) If $f \in L$ and $\mu(\mathbf{M} f=0)>0$, then (22) holds for each $g \in L$ such that $g=f$ on $(\mathbf{M} f>0)$ and $g \leq f$ on $(\mathbf{M} f=0)$.

Since this theorem can be proved as in [3], we omit its proof here.

\section{ACKNOWLEDGEMENT}

The research was supported by the CNR-NATO grant of Italy No. 217.35 S. 


\section{REFERENCES}

1. L. Ephremidze, On the uniqueness of maximal functions. Georgian Math. J. 3(1996), $49-52$.

2. L. Ephremidze, On the uniqueness of maximal operators for ergodic flows. Rev. Mat. Complut. 15(2002), No. 1, 75-84.

3. L. Ephremidze, On the uniqueness of the ergodic maximal function. Fund. Math. 174(2002), No. 3, 217-228.

4. R. L. Jones, On the uniqueness of the ergodic maximal function. Proc. Amer. Math. Soc. 132(2004), No. 4, 1087-1090.

5. P. R. Halmos, Lectures on ergodic theory. Publications of the Mathematical Society of Japan, No. 3. The Mathematical Society of Japan, 1956.

6. K. E. Petersen, Ergodic theory. Cambridge Studies in Advanced Mathematics, 2. Cambridge University Press, Cambridge, 1983.

7. P. Sjogren, How to recognize a discrete maximal function. Indiana Univ. Math. J. 37(1988), No. 4, 891-898.

(Received 29.09.2004)

Author's address:

A. Razmadze Mathematical Institute

Georgian Academy of Sciences

1, M. Aleksidze St., Tbilisi 0193

Georgia

E-mail: lasha@rmi.acnet.ge 